\title{
Reconsidering metasurface lasers
}

\author{
Dandan Wen ${ }^{1}$, Jasper J. Cadusch ${ }^{1}$, Zhiqiang Fang ${ }^{2}$ and Kenneth B. Crozier, ${ }^{1,3,4 *}$
}

1 Department of Electrical and Electronic Engineering, University of Melbourne, Victoria 3010, Australia

2 International Collaborative Laboratory of 2D Materials for Optoelectronics Science and Technology,

Shenzhen University, Shenzhen 518060, China

3 School of Physics, University of Melbourne, Victoria 3010, Australia

4 Australian Research Council (ARC) Centre of Excellence for Transformative Meta-Optical Systems,

University of Melbourne, Victoria 3010, Australia

*Email:kcrozier@unimelb.edu.au

Metasurface based orbital angular momentum (OAM) generation has attracted considerable attention recently $1,2,3,4,5,6,7$. The recently published article "High-purity orbital angular momentum states from a visible metasurface laser" presents a very neat method for orbital angular momentum (OAM) optical state generation ${ }^{8}$. However, we suspect that the explanation of the operating principle provided by the authors is incorrect or incomplete. We anticipate that this could hamper future progress in not only metasurface lasers but also metasurfaces in general if left uncorrected.

As we understand it, the authors intend to generate a resonant cavity mode, which should have the same state after one round trip within the cavity. To explain this point, the authors state that "Starting as $\mid H$, $0>$ (horizontally polarized and no OAM), it is converted to the desired combination within the laser, say $\cos \theta\left|H, l_{1}>+\sin \theta\right| V, l_{2}>\ldots$ On reflection from the output coupler mirror back to the J-plate, the state is $\cos \theta\left|H,-l_{1}>+\sin \theta\right| V,-l_{2}>$. From the reciprocity of the metasurface, the initial state $\mid H, 0>$ is regained, which then returns through the polarizer to the KTP crystal'. We agree that, after the reflection from the output coupler mirror, the state changes to $\cos \theta\left|H,-l_{1}>+\sin \theta\right| V,-l_{2}>$. However, the initial state $\mid \mathrm{H}, 0>$ cannot be regained by what the authors refer to as "the reciprocity of the metasurface" because the presence of the J-plate within the cavity will change the state to $\cos \theta \mid H$, $2 l_{1}>+\sin \theta \mid V,-2 l_{2}>$, rather than back to the initial OAM state. We explain our point below.

Throughout this Comment, we take JP1(in the nomenclature of the authors) as an example upon which all discussions are based. We also assume that the fast axis of the J-plate is along the vertical direction $\left(\varphi=90^{\circ}\right.$ in Fig. 1). We take the polarization of the incident light as being horizontal. Therefore, we do not need to consider the phase retardance between the fast and slow axes of the waveplate. An OAM beam with $l=1$ will be generated according to the JP1 design. After the horizontally polarized beam with no OAM $\left(E_{0}\right)$ is transformed by the J-plate (from area A to area B in Fig.1), the field will have the following form in the $x y z$ coordinate system. Note that, as discussed, the field is $x$-polarized.

$$
E_{1}=E_{0} \exp [i * \varphi(x, y)]
$$


where we have $z=0$ here and $\varphi(x, y)$ represents the azimuthal angle of the point $(x, y)$. The beam travels the distance $\Delta z$ in area $\mathrm{B}$ and approaches the mirror. Then the field becomes

$$
E_{2}=E_{0} \exp [i * \varphi(x, y)+i k \Delta z]
$$

Where $k=2 \pi / \lambda$. The expression for $E_{2}$ is still expressed in the $x y z$ coordinate system. After $E_{2}$ is reflected by the mirror, the propagation directed is reversed (from $z$ to $z^{\prime}$ direction). We thus use the $x^{\prime} y^{\prime} z^{\prime}$ coordinate system. The azimuth angle $\varphi^{\prime}\left(x^{\prime}, y^{\prime}\right)$ has the following relationship with $\varphi(x, y)$

$$
\varphi(x, y)=\pi-\varphi^{\prime}\left(x^{\prime}, y^{\prime}\right)
$$

This can be easily verified, for example, we have $\varphi=45^{\circ}$ as shown in the $x y z$ coordinate system which corresponds to $\varphi^{\prime}=135^{\circ}$ in the new $x^{\prime} y^{\prime} z^{\prime}$ coordinates (Fig.1). We rewrite $E_{2}$ in the $x^{\prime} y^{\prime} z^{\prime}$ system and find

$$
E_{2}=-E_{0} \exp \left[-i * \varphi^{\prime}\left(x^{\prime}, y^{\prime}\right)+i \pi+i k \Delta z\right]
$$

This demonstrates that we have an OAM beam with $l=-1$ in the $x^{\prime} y^{\prime} z^{\prime}$ coordinate system. This agrees, so far, with the authors' description. On the return trip, the phase change caused by the J-plate is also rewritten in the $x^{\prime} y^{\prime} z^{\prime}$ coordinates as

$$
\Phi\left(x^{\prime}, y^{\prime}\right)=\pi-\varphi^{\prime}\left(x^{\prime}, y^{\prime}\right)
$$

The field after passing through the J plate again (from area B to area A) is thus given by:

$$
\begin{aligned}
E_{3}=E_{2} e^{i k \Delta z} e^{i \Phi\left(x^{\prime}, y^{\prime}\right)} & =-E_{0} \exp \left[-i * 2 \varphi^{\prime}\left(x^{\prime}, y^{\prime}\right)+i 2 \pi+i 2 k \Delta z\right] \\
& =-E_{0} \exp \left[-i * 2 \varphi^{\prime}\left(x^{\prime}, y^{\prime}\right)\right] * \exp [2 i(\pi+k \Delta z)]
\end{aligned}
$$

We abandon the term $\exp [2 i(\pi+k \Delta z)]$ since it does not depend on lateral coordinates $\left(x^{\prime}, y^{\prime}\right)$. Therefore, this field $\left(E_{3}\right)$ has a topological charge of $\mathbf{- 2}$, instead of 0 as mentioned by the authors. Since the J-plate works in a manner of traditional spiral phase element here, our point is also consistent with the analysis of previous works ${ }^{6,9}$. This explanation also applies when the fast axis of the J-plate is aligned along other angles.

In summary, we conclude that the explanation of the operating principle presented in the original paper is incorrect or incomplete. That paper however reported experimental results, so we anticipate that there must be another mechanism at play that allows the round trip condition to be met and the metasurface laser to operate. 


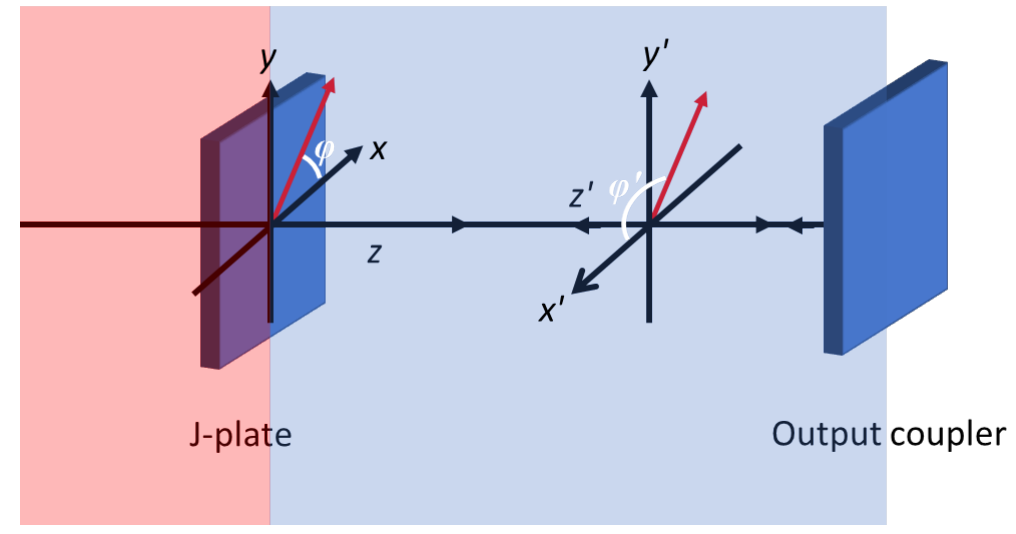

\section{Competing interests}

The authors declare no competing interests.

\section{References}

1. Li G, Kang M, Chen S, Zhang S, Pun EY-B, Cheah KW, et al. Spin-Enabled Plasmonic Metasurfaces for Manipulating Orbital Angular Momentum of Light. Nano Lett. 13, 41484151 (2013).

2. Pu M, Li X, Ma X, Wang Y, Zhao Z, Wang C, et al. Catenary optics for achromatic generation of perfect optical angular momentum. Sci. Adv. 1, e1500396 (2015).

3. Yang Y, Wang W, Moitra P, Kravchenko II, Briggs DP, Valentine J. Dielectric MetaReflectarray for Broadband Linear Polarization Conversion and Optical Vortex Generation. Nano Lett. 14, 1394-1399 (2014).

4. Karimi E, Schulz SA, De Leon I, Qassim H, Upham J, Boyd RW. Generating optical orbital angular momentum at visible wavelengths using a plasmonic metasurface. Light Sci. Appl. 3, e167 (2014).

5. Huang C, Zhang C, Xiao S, Wang Y, Fan Y, Liu Y, et al. Ultrafast control of vortex microlasers. Science 367, 1018-1021 (2020).

6. Maguid E, Chriki R, Yannai M, Kleiner V, Hasman E, Friesem AA, et al. Topologically Controlled Intracavity Laser Modes Based on Pancharatnam-Berry Phase. ACS Photonics $\mathbf{5}$, 1817-1821 (2018). 
7. Stellinga D, Pietrzyk ME, Glackin JME, Wang Y, Bansal AK, Turnbull GA, et al. An Organic Vortex Laser. ACS Nano 12, 2389-2394 (2018).

8. Sroor H, Huang Y-W, Sephton B, Naidoo D, Vallés A, Ginis V, et al. High-purity orbital angular momentum states from a visible metasurface laser. Nat. Photonics 1-6 (2020).

9. Oron R, Davidson N, Friesem AA, Hasman E, Wolf E. Transverse mode shaping and selection in laser resonators. Prog. Opt 42, 325-386 (2001). 


\section{University Library}

\section{- M M I E R R V A gateway to Melbourne's research publications}

Minerva Access is the Institutional Repository of The University of Melbourne

Author/s:

Wen, D;Cadusch, JJ;Fang, Z;Crozier, KB

Title:

Reconsidering metasurface lasers

Date:

2021-05-01

Citation:

Wen, D., Cadusch, J. J., Fang, Z. \& Crozier, K. B. (2021). Reconsidering metasurface lasers. NATURE PHOTONICS, 15 (5), pp.337-338. https://doi.org/10.1038/s41566-021-00806-x.

Persistent Link:

http://hdl.handle.net/11343/294847 